\title{
Hyperplane arrangements in negatively curved manifolds and relative hyperbolicity
}

\author{
Igor Belegradek and G. Christopher Hruska*
}

\begin{abstract}
We show that certain aspherical manifolds arising from hyperplane arrangements in negatively curved manifolds have relatively hyperbolic fundamental group.
\end{abstract}

Mathematics Subject Classification (2010). 20F65.

Keywords. Relatively hyperbolic group, hyperplane arrangement, simplicial volume, negatively curved manifold.

\section{Introduction}

Let $M$ be a connected, complete, finite volume Riemannian $n$-manifold of sectional curvature satisfying $\varkappa \leq \sec (M) \leq-1$ for some constant $\varkappa$, and let $S \subset M$ be a subset whose preimage to the universal cover of $M$ is the union of a locally finite family $\mathscr{D}$ of hyperplanes, where a hyperplane is a complete totally geodesic submanifold of codimension two.

In this paper we study the fundamental group of $M \backslash S$, which clearly surjects onto $\pi_{1}(M)$ as $S$ has codimension two. One may think of $\pi_{1}(M \backslash S)$ as an "overlattice", i.e. a group that comes with a natural surjection onto a lattice (cf. [29], page 192). This paper explores to what extent $\pi_{1}(M \backslash S)$ inherits some rigidity properties of lattices, and our approach is to find conditions on $M, S$ implying that $\pi_{1}(M \backslash S)$ is non-elementary relatively hyperbolic. In recent years many powerful techniques have been developed to better understand relatively hyperbolic groups, and this paper allows to apply the techniques to studying certain $M \backslash S$ 's.

Set $m=\left[\frac{n}{2}\right]$. Following Allcock [2] we call $\mathcal{D}$ normal if hyperplanes in $\mathscr{D}$ are either disjoint or orthogonal, and if for any point $p$ lying on hyperplanes $h_{1}, \ldots, h_{k}$ in $\mathcal{D}$ there is a linear isomorphism of the tangent space at $p$ onto $\mathbb{R}^{n-2 m} \times \mathbb{C}^{m}$ that maps the tangent space to each $h_{i}$ to the product of $\mathbb{R}^{n-2 m}$ with a coordinate hyperplane in $\mathbb{C}^{m}$. We call $S$ normal if $\mathcal{D}$ is normal. Many examples of normal $S \subset M$ are known when $M$ is real hyperbolic or complex hyperbolic.

\footnotetext{
*Supported by NSF \# DMS-0804038 (Belegradek) and \#DMS-0808809 (Hruska).
} 
Gromov stated in [28], Section 4.4A, and Allcock proved in [2] that if $S$ is normal in $M$, then the metric completion of the universal Riemannian cover of $M \backslash S$ is CAT(-1), and Allcock furthermore used this to show that the manifold $M \backslash S$ is aspherical (i.e. its universal cover is contractible); thus most if not all topological information about $M \backslash S$ is encoded in its fundamental group.

We work with the combinatorial definition of a relatively hyperbolic group due to Bowditch [15], Definition 2, and call a subgroup of relatively hyperbolic group nonelementary unless it is finite, virtually- $\mathbb{Z}$, or lies in a peripheral subgroup. Building on ideas of Bowditch [15], we prove:

Theorem 1.1. Suppose that $M$ contains a closed, locally convex subset $V$ such that $M \backslash V$ is nonempty and precompact in $M$, and $S$ lies in the interior of $V$. If $S$ is normal in $M$, then $\pi_{1}(M \backslash S)$ is non-elementary relatively hyperbolic, where peripheral subgroups are the fundamental groups of components of $V \backslash S$; furthermore, each component of $V \backslash S$ is aspherical, and its inclusion into $M \backslash S$ is $\pi_{1}$-injective.

To apply this theorem one needs to find $V$, and e.g. if $M$ is compact, then a natural candidate for $V$ would be (a sufficiently small $\varepsilon$-neighborhood of) the smallest locally convex subset of $M$ that contains $S$; if this $\varepsilon$-neighborhood is a proper subset of $M$, then Theorem 1.1 applies. The same is true for non-compact $M$ except that we also need to require that $V$ contains all cusps.

The simplest picture emerges when $S$ is a compact smooth submanifold of $M$. Then $S$ is normal, and we can take $V$ to be the union of a small $\varepsilon$-tubular neighborhood of $S$ and sufficiently small disjoint cusp neighborhoods of $M$, which implies the following.

Corollary 1.2. If $S$ is a compact smooth submanifold of $M$, then the group $\pi_{1}(M \backslash S)$ is non-elementary relatively hyperbolic, where the peripheral subgroups are either the fundamental groups of circle bundle over components of $S$, or the fundamental groups of compact infranilmanifolds which are cusp cross-sections of $M$.

Special cases of Corollary 1.2 were previously proved in [9] (when $M$ is real hyperbolic) and [8] (when $M$ and $S$ are complex hyperbolic). The methods used in [8], [9] involve delicate warped product computations that are entirely different from the approach adopted in the present article.

Corollary 1.3. If $n \geq 3$ and $S$ is a compact smooth submanifold of $M$, then

- $\pi_{1}(M \backslash S)$ has solvable word and conjugacy problems, has finite asymptotic dimension and rapid decay property, is co-Hopf and residually hyperbolic, has finite outer automorphism group;

- if $\pi_{1}(M \backslash S)$ splits nontrivially as an amalgamated product or HNN-extension over a subgroup $K$, then $K$ contains a non-abelian free subgroup; 
- if $M$ is compact, then $\pi_{1}(M \backslash S)$ is biautomatic and satisfies Strong Tits Alternative.

As in Theorem 1.2 of [9], one also gets a Mostow type rigidity theorem, namely if $S_{i}$ is compact and normal in $M_{i}$ for $i=1,2$, and if each $M_{i}$ is locally symmetric of dimension $>2$, then any homotopy equivalence $M_{1} \backslash S_{1} \rightarrow M_{2} \backslash S_{2}$ is homotopic to the restriction of an isometry $M_{1} \rightarrow M_{2}$ that takes $S_{1}$ to $S_{2}$. This is only new in the "exceptional" case when one of the $\left(S_{i}, M_{i}\right)$ 's is locally modelled on a totally real plane in the complex hyperbolic plane.

Most results on structure and properties of relatively hyperbolic groups are relative in nature, i.e., properties of the peripheral subgroups are inherited by the ambient relatively hyperbolic group. In general, the peripheral subgroups in Theorem 1.1 are similar to the group $\pi_{1}(M \backslash S)$ and little is known about them. The reason relative hyperbolicity gives so much information for compact embedded $S$ is that it is possible to choose $V$ such that the topology of $V \backslash S$ is easy to understand.

The main result of this paper ensures the existence of $V$ with an easy to understand $V \backslash S$, provided $S$ is normal and "sparse". To make this precise it helps to introduce the following notations. Recall that if $M$ is non-compact, then each end of $M$ has an arbitrary small, closed, connected neighborhood which is called a cusp; its preimage to the universal cover of $M$ is the union of a family of disjoint horoballs. Fix a collection of disjoint cusps of $M$, one for each end, and let $Q$ be their union, and let $\mathscr{H}$ be the corresponding family of pairwise disjoint horoballs. We assume that either $S$ and $Q$ are disjoint, or $S$ intersects $\partial Q$ orthogonally, which can be always arranged by choosing $Q$ sufficiently small, as noted in the beginning of Section 3 .

We say that $\{S, Q\}$ is $r$-sparse if for any disjoint sets $A, B \in \mathscr{D} \cup \mathscr{H}$ that contain points $a \in A$ and $b \in B$ with $\operatorname{dist}(a, b)<r$, one has $a, b \in C$ for some $C \in \mathscr{H}$. Since $A \cap B=\varnothing$, and since all horoballs in $\mathscr{H}$ are disjoint it then follows that $A, B$ are hyperplanes in $\mathscr{D}$ that are asymptotic to the center of the horoball $C$. In other words, $r$-sparse means that the only way two disjoint convex sets in $\mathscr{D} \cup \mathcal{H}$ can come within distance $r$ is inside a horoball from $\mathscr{H}$. We say that $\{\mathscr{D}, \mathscr{H}\}$ is $r$-sparse if $\{S, Q\}$ is $r$-sparse. Compactness of $M \backslash \operatorname{Int}(Q)$ implies that any $\{S, Q\}$ is $r$-sparse for each sufficiently small $r$.

All this holds verbatim when $M$ is compact by letting $Q=\varnothing$. In this case the definition of sparseness simplifies: $\{S, \varnothing\}$ is $r$-sparse if and only if the distance between any two disjoint hyperplanes in $\mathscr{D}$ is $\geq r$. We prove:

Theorem 1.4. There is a positive constant $r(\varkappa, n)$ such that if $S$ is normal and $\{S, Q\}$ is $r(\varkappa, n)$-sparse, then $M \backslash S$ is diffeomorphic to the interior of a compact manifold $N$ such that

(1) each component of $\partial N$ is aspherical;

(2) the inclusion $\partial N \hookrightarrow N$ is $\pi_{1}$-injective;

(3) $\pi_{1}(N)$ is non-elementary relatively hyperbolic; 
(4) conjugacy classes of peripheral subgroups bijectively correspond to the fundamental groups of components of $\partial N$;

(5) if $n \geq 3$ and $\pi_{1}(N)$ splits nontrivially as an amalgamated product or $H N N$ extension over a subgroup $K$, then $K$ contains a non-abelian free subgroup;

(6) if $n \geq 3$ and $S$ is compact, then $\operatorname{Out}\left(\pi_{1}(N)\right)$ is finite;

(7) if $n \geq 3$, then $\pi_{1}(N)$ is co-Hopf;

(8) if $n=4$, then $\operatorname{Out}\left(\pi_{1}(N)\right)$ is finite, and furthermore $\pi_{1}(N)$ has solvable word and conjugacy problems, has finite asymptotic dimension, and is residually hyperbolic.

Most likely, (6) still holds for non-compact $S$, but our proof does not apply. In (5) we actually show that $K$ must be non-elementary in the relatively hyperbolic group structure given by (3)-(4). Part (8) hinges on various known results about 3-manifold groups, appearing here as peripheral subgroups of $\pi_{1}(N)$.

The manifold $N$ is constructed in a canonical way, namely, shrinking along the rays orthogonal to $\partial Q$ gives a diffeomorphism of $M \backslash S$ onto $M \backslash(Q \cup S)$, and the latter is the interior of a compact manifold $N$, obtained by removing from $M$ the interior of a regular neighborhood of $Q \cup S$.

It is easy to construct examples to which Theorem 1.4 applies, e.g. if $\pi_{1}(M)$ is residually finite, then any $\pi_{1}(M)$-invariant locally finite normal hyperplane arrangement in the universal cover can be thinned out by passing to a finite index subgroup and removing orbits of some hyperplanes/horoballs to ensure sparseness.

We do not know if Theorem 1.4 applies to any of the "natural" examples such as arrangements coming from Lorentzian lattices in [2]. A basic difficulty is that the constant $r(\varkappa, n)$ arising in the proof of Theorem 1.4 seems much larger than the sparseness constants for the "natural" examples; we hope to address this in future work.

To prove Theorem 1.4 we find $V$ as in Theorem 1.1 with $V \backslash(S \cup Q)$ homotopy equivalent to the boundary of a regular neighborhood of $Q \cup S$ in $M$. This hinges on the result of Bowditch [14] that in a negatively pinched Hadamard manifold, any quasiconvex subset is within bounded distance to its convex hull; quasiconvexity follows from sparseness. This result in [14] depends on a delicate construction in [5], which both contribute to the size of $r(\varkappa, n)$.

A few properties of non-elementary relatively hyperbolic groups hold even if nothing is known about peripheral subgroups, e.g. each subnormal subgroup of a nonelementary relatively hyperbolic group is non-elementary (as follows from a standard argument using its action on the ideal boundary). In particular, each subnormal subgroup of a non-elementary relatively hyperbolic group contains a non-abelian free subgroup [41] and has infinite dimensional second bounded cohomology [26].

In another direction it was proved in [9] that if a non-elementary relatively hyperbolic group is isomorphic to a lattice in a virtually connected Lie group, then the lattice has real rank one. The latter cannot happen for $\pi_{1}(M \backslash S)$, as long as $S$ is normal and $n>3$, due to the following. 
Theorem 1.5. If $S$ is normal and $n>3$, then $\pi_{1}(M \backslash S)$ has a nontrivial element whose centralizer contains a non-abelian free subgroup, and in particular, $\pi_{1}(M \backslash S)$ is not isomorphic to a discrete isometry group of a Hadamard manifold of pinched negative curvature.

Theorem 1.5 fails if $n \leq 3$ in which case $M \backslash S$ is often hyperbolizable. For normal $S$ and $n>3$, it is likely that $\pi_{1}(M \backslash S)$ is never isomorphic to a lattice in a virtually connected Lie group; this was proved in [3] in the case when $M, S$ are complex hyperbolic, and Theorem 1.5 proves it in case $\pi_{1}(M \backslash S)$ is non-elementary relatively hyperbolic.

It is interesting that in some cases $M \backslash S$ admits a complete finite volume metric of $\sec \in[-1,0)$, see [1], [9], [8], yet the existence of such metric does not (seem to) have significant group theoretic implications.

To prove finiteness of $\operatorname{Out}\left(\pi_{1}(M \backslash S)\right)$ in Theorem 1.4(6) we use another property of relatively hyperbolic groups that assumes nothing about peripheral subgroups, and which is an application of recent work of Mineyev-Yaman [33]:

Proposition 1.6. Let $L$ be a closed aspherical manifold such that $\pi_{1}(L)$ is nonelementary relatively hyperbolic, then the simplicial volume $\|L\|$ is positive.

The proof of Theorem 1.1 is inspired by arguments of Bowditch [15], where it is implicit that if $M$ contains a closed, locally convex subset $V$ such that $M \backslash V$ is nonempty and precompact in $M$, then $\pi_{1}(M)$ is hyperbolic relative to the fundamental groups of components of $V$ (see Remark 4.6). Combining this statement with a result of Mineyev-Yaman [33] gives the following.

Theorem 1.7. Suppose that $M$ contains a closed, locally convex subset $V$ such that $M \backslash V$ is nonempty and precompact in $M$. If $S \subset \operatorname{Int}(V)$, then the simplicial volume $\|M \backslash S\|$ is nonzero.

Gromov introduced the notion of simplicial volume $\|U\|$ of a manifold $U$ in his seminal work [27]. Simplicial volume takes values in $[0, \infty]$ and is a proper homotopy invariant of the manifold $U$. Nonvanishing of $\|U\|$ has various geometric and topological consequences, e.g.,

- any complete Riemannian metric on $U$ with $\operatorname{Ric}(U) \geq-(n-1)$ has the lower volume bound $\|U\| \leq c_{n} \operatorname{Vol}(U)$, see page 12 in [27];

- if $U$ is the interior of a compact manifold, then $\|U\|>0$ implies that $U$ admits no proper self-maps of degree $>1$, see page 8 in [27].

These two facts hold even if $\|U\|$ is infinite. It follows from [27], page 59, that $\|M \backslash S\|$ is finite provided $M$ is compact and $S$ is normal; this result is used in the proof of Theorem 1.4(6). By contrast, if $S$ is non-compact and $n=3$, then $\|M \backslash S\|$ is always infinite, because $M \backslash S$ is the interior of a compact manifold whose boundary has nonzero simplicial volume. 
The structure of the paper is as follows. In Section 2 we give a general criterion for relative hyperbolicity of a group acting on a CAT $(-1)$ space. Section 3 is a list of notations and standing assumptions. In Section 4 we establish various preliminary results culminating in Theorem 4.4 , which is an orbifold version of Theorem 1.1. Note that Corollary 1.2 is immediate from Theorems 1.1 and the structure of finite volume manifolds of pinched negatively curvature. An orbifold version of Theorem 1.4 is proved in Section 5, while Section 6 contains proofs of Corollary 1.3, Theorem 1.5, Proposition 1.6, and Theorem 1.7. In the Appendix we collect some facts on CAT $(-1)$ spaces that we could not find in the literature.

Acknowledgments. Belegradek is grateful to Ian Agol, Daniel Allcock, Greg Kuperberg, and Henry Wilton for helpful communications.

\section{A criterion for proving relative hyperbolicity}

In [15] Bowditch showed that a group is relatively hyperbolic if and only if it acts on fine, connected, hyperbolic graph with finite quotient and finite edge stabilizers. Here a graph is given the path-metric in which each edge is isometric to the unit interval, and if the path-metric is Gromov hyperbolic, the graph is called hyperbolic. A graph is called fine if for each $n$ each edge lies in only finitely many circuits of length $<n$, where a circuit is an embedded closed path. A family of subsets in a metric space is called $r$-separated if the distance between any two subsets in the family is $\geq r$ where $r \in \mathbb{R}$. We prove:

Theorem 2.1. Let $X$ be a complete CAT $(-1)$ space, and let $\varepsilon>0$. Suppose that there exists a subgroup $H \leq \operatorname{Iso}(X)$ and a $H$-invariant family $\mathcal{A}=\left\{A_{i}\right\}$ of $\varepsilon$-separated closed convex subsets of $X$ such that $X \backslash \bigcup_{i} \operatorname{Int}\left(A_{i}\right)$ is locally compact, and $H$-action on $X \backslash \bigcup_{i} \operatorname{Int}\left(A_{i}\right)$ is properly discontinuous and cocompact. If $H_{i}$ is the stabilizer of $A_{i}$ in $H$, then $H$ is hyperbolic relative to $\left\{H_{i}\right\}$.

Remark 2.2. In this paper we apply Theorem 2.1 to spaces $X$ that are not proper. If $X$ is proper, Theorem 2.1 is essentially due to Bowditch, e.g. it follows from 7.12-7.13 in [15] and (the elementary) Lemma A.3 below.

Proof. By Lemma A.1 below for every distinct $A_{i}, A_{j}$ there is a unique segment $\left[a_{i j}, a_{j i}\right]$ that realizes the distance between $A_{i}, A_{j}$ where $a_{k l} \in A_{k}$.

Given $u>0$, let $\Gamma_{u}$ be the $u$-nerve of $\left\{A_{k}\right\}$, i.e., the graph with vertex set $\mathcal{A}$ and $A_{i}, A_{j}$ are joined by an edge if and only if $d\left(a_{i j}, a_{j i}\right) \leq u$. Since $H$ acts cocompactly on $X \backslash \bigcup_{i} \operatorname{Int}\left(A_{i}\right)$, the graph $\Gamma_{u}$ is connected, provided $u$ is large enough. Fix such $u$ and give $\Gamma_{u}$ a path-metric with edges of length 1. By [15], Proposition 7.12, $\Gamma_{u}$ is a hyperbolic metric space in this path-metric. It is easy to see that $\Gamma_{u}$ need not be fine. Below we replace $\Gamma_{u}$ by a subgraph that is fine. 
Let $\Gamma_{u}^{\prime}$ be the subgraph of $\Gamma_{u}$ with the same vertices in which $A_{i}, A_{j}$ are joined by an edge if and only if $\left[a_{i j}, a_{j i}\right] \cap A_{k}=\emptyset$ for each $k \notin\{i, j\}$. The graph $\Gamma_{u}^{\prime}$ is $H$-invariant, and since $H$ acts properly discontinuously and cocompactly on $X \backslash \bigcup_{i} \operatorname{Int}\left(A_{i}\right)$, the quotient graph $\Gamma_{u} / H$ is finite, and hence so is $\Gamma_{u}^{\prime} / H$. Moreover, uniqueness of $\left[a_{i j}, a_{j i}\right]$ implies that edge stabilizers are finite.

Lemma 2.3. If two vertices of $\Gamma_{u}$ are joined by an edge, then they are joined by a path of length $\leq \frac{u}{\varepsilon}$ that lies in $\Gamma_{u}^{\prime}$.

Proof. Indeed, suppose $A_{i}, A_{j}$ are joined by an edge of $\Gamma_{u}$, so that $\left[a_{i j}, a_{j i}\right]$ has length $\leq u$. If $\left[a_{i j}, a_{j i}\right]$ passes through some $A_{m}$, then $\operatorname{dist}\left(A_{i}, A_{j}\right) \geq \operatorname{dist}\left(A_{i}, A_{m}\right)+$ $\operatorname{dist}\left(A_{m}, A_{j}\right)$. Since the $A_{k}$ 's are $\varepsilon$-separated, $\operatorname{dist}\left(A_{i}, A_{m}\right)$ and $\operatorname{dist}\left(A_{m}, A_{j}\right)$ are $\leq u-\varepsilon$. Repeating the process for each pair, we note that after each step they become closer by $\varepsilon$, so the procedure terminates at a finite sequence $A_{i}, \ldots, A_{j}$ such that the sum of lengths between adjacent sets is $\leq u$. Hence $A_{i}, A_{j}$ are joined by a path in $\Gamma_{u}^{\prime}$ of length $\leq \frac{u}{\varepsilon}$.

Thus $\Gamma_{u}^{\prime}$ is connected, and in the path-metric induced from $\Gamma_{u}$ the graph $\Gamma_{u}^{\prime}$ is quasi-isometric to $\Gamma_{u}$, in particular, $\Gamma_{u}^{\prime}$ is hyperbolic.

Towards proving that $\Gamma_{u}^{\prime}$ is fine, recall that a family of subsets $\left\{Q_{i}\right\}$ in a metric space is said to have bounded penetration if there is a function $D(\rho)$ such that for each $k \neq j$ the intersection of the $\rho$-neighborhoods of $Q_{k}, Q_{j}$ has diameter $\leq D(\rho)$. By Lemma A.3 since the family $\left\{A_{k}\right\}$ is $\varepsilon$-separated, it has bounded penetration.

Consider an arbitrary circuit in $\Gamma_{u}^{\prime}$ of length $n$. Represent it by a piecewise geodesic loop $\gamma$ in $X$ written as $\alpha_{1} \cup \beta_{1} \cup \cdots \cup \alpha_{n} \cup \beta_{n}$ where $\alpha_{i}$ is a geodesic segment in $A_{i}$ with endpoints in $\partial A_{i}$ and $\beta_{i}$ is the segment $\left[a_{i}, a_{i+1}\right]$ joining $\partial A_{i}$ to $\partial A_{i+1}$. Since $\left\{A_{k}\right\}$ has bounded penetration, the length of $\gamma$ is bounded above by a linear function of $n$ as proved by Bowditch in a comment right before Lemma 7.13 in [15]. (For completeness we outline his argument. That $\mathcal{A}$ has bounded penetration implies that $\gamma$ has bounded backtracking, i.e., any two geodesic segments that form $\gamma$ only travel together for a bounded amount of time. This is trivially true for $\beta_{i}$ 's as they have length $\leq u$, and for $\alpha_{i}$ 's this follows from bounded penetration. Now a linear bound on the length of $\gamma$ can be obtained from Proposition 5.7 of [15], which is essentially Proposition 7.3.4 of [12] whose proof is fairly long. For the purposes of this paper a linear bound is not important, indeed any bound would do, and an easier argument in [15] (Corollary 7.2) gives a quadratic bound, i.e. the length of $\gamma$ is bounded above by a quadratic function of $n$.)

Now we can finish the proof that $\Gamma_{u}^{\prime}$ is fine. The subpath of $\gamma$ given as $\gamma_{1}:=$ $\beta_{1} \cup \alpha_{2} \cup \cdots \cup \beta_{n}$ lies outside $A_{1}$, so the orthogonal projection $X \rightarrow A_{1}$ maps $\gamma_{1}$ to a path $\bar{\gamma}_{1}$ in $\partial A_{1}$ joining the endpoints of $\alpha_{1}$ (where the boundary of a subset $Z$ is defined by $\partial Z:=\bar{Z}-\operatorname{Int}(Z)$ ). Since the projection is distance-nonincreasing, the distance between the endpoints of $\alpha_{1}$ in the path-metric on $\partial A_{1}$ induced from $X$ is bounded by the same linear function of $n$. Applying this argument to each $\alpha_{i}$, we 
see that $\alpha_{i}$ can be replaced by a path in $\partial A_{i}$ with the same endpoints, giving the new loop $\gamma^{\prime}:=\bar{\gamma}_{1} \cup \beta_{1} \cup \cdots \cup \bar{\gamma}_{n} \cup \beta_{n}$ in $X \backslash \bigcup_{i} \operatorname{Int}\left(A_{i}\right)$ whose length is bounded by a quadratic function of $n$. The space $X \backslash \bigcup_{i} \operatorname{Int}\left(A_{i}\right)$, equipped with the path metric induced from $X$, is proper by Hopf-Rinow because it is locally compact and complete. In this metric the family $\left\{\partial A_{k}\right\}$ is locally finite because it is $\varepsilon$-separated, and hence only finitely many $\partial A_{k}$ 's can be visited by $\gamma^{\prime}$ as above provided it contains $\beta_{1}$. Thus the number of circuits of length $n$ in $\Gamma_{u}^{\prime}$ that contain a given edge is finite, so $\Gamma_{u}^{\prime}$ is fine. This completes the proof of Theorem 2.1.

Remark 2.4. The proof of Theorem 2.1 goes through with minor modifications when $X$ is $\delta$-hyperbolic provided the $A_{i}$ 's are $r$-separated with $r \gg \delta$.

\section{Notations and standing assumptions}

In the introduction we focused on manifolds, yet all "natural" examples we know are orbifolds, so we work equivariantly in the universal cover and allow lattices with torsion; this necessitates a slight change in notations. Similarly, it would be easier to deal with compact orbifolds but many "natural" examples are non-compact.

Let $Y$ be a complete simply-connected Riemannian manifold of sectional curvatures within $[\varkappa,-1]$, for some constant $\varkappa \leq-1$, and let $G$ be discrete isometry group of $Y$ such that the orbifold $Y / G$ has finite volume.

Let $\mathscr{D}$ be a locally finite $G$-invariant family of hyperplanes in $Y$ (recall that hyperplanes are complete totally geodesic submanifolds of codimension two). Let $D$ denote the union of the hyperplanes in $\mathscr{D}$.

Suppose that $Y$ contains a closed, $G$-invariant, locally convex subset $C$ with nonempty $C^{1}$-smooth boundary $\partial C$ such that $D \subset \operatorname{Int}(C)$, and $G$ acts cocompactly on $Y \backslash \operatorname{Int}(C)$. Thus $\partial C / G$ is a compact, and therefore, $\partial C$ has a positive normal injectivity radius.

Remark 3.1. All the assumptions on $C$ are crucial except for " $\partial C$ is $C^{1 \text { " which }}$ simplifies some matters, and causes no loss of generality. Indeed, if $C$ is as in the previous paragraph except that $\partial C$ is not $C^{1}$, then $C$ is a (codimension zero) topological submanifold with possibly non-smooth boundary ([19], Theorem 1.6). Now according to Theorem 1.2 of [40] or Lemma 5 of [37] the distance function to $C$ is $C^{1}$ near $\partial C$. Since $C$ is $G$-invariant and $G$ acts cocompactly on $\partial C$, any sufficiently small $\varepsilon$-neighborhood of $C$ has $C^{1}$-boundary, and since the curvature is nonpositive, the $\varepsilon$-neighborhood of $C$ is locally convex, so by replacing $C$ with its $\varepsilon$-neighborhood we may assume its boundary is $C^{1}$.

The orbifold $Y / G$ is the union of a compact set, and finitely many cusps whose preimage in $Y$ is a $G$-invariant family $\mathscr{H}$ of disjoint closed horoballs, see [14], Proposition 6.6. 
Suppose that every horoball in $\mathscr{H}$ is either disjoint from $D$, or intersects $D$ orthogonally. (This can be always arranged by choosing horoballs in $\mathscr{H}$ sufficiently small, for otherwise since horoballs fall into finitely many $G$-equivalence types, there is a sequence of concentric horoballs $B_{i}$ that Hausdorff converges to their common center $z$, and a sequence of hyperplanes $h_{i}$ such that the intersection $h_{i} \cap \partial B_{i}$ is not orthogonal. Acting by $\operatorname{Stab}_{G}(z)$, we may assume each $h_{i}$ intersects a compact fundamental domain for the $\operatorname{Stab}_{G}(z)$-action on $\partial B_{0}$. By local finiteness of $\left\{h_{i}\right\}$, we can find $h_{i_{0}}$ that intersects each $B_{i}$, hence $z$ lies at infinity of $h_{i_{0}}$ implying that $h_{i_{0}}$ is orthogonal to the boundary of any horoball concentric to $B_{0}$, which contradicts the assumption.)

Let $Y_{0}:=Y \backslash D$, and let $p_{0}: X_{0} \rightarrow Y_{0}$ be the universal Riemannian covering. Let $X$ be the metric completion of $X_{0}$. Since $p_{0}$ is a local isometry, it is distance nonincreasing, so it maps Cauchy sequences to Cauchy sequences, and hence extends to a continuous map of metric completions $p: X \rightarrow Y$, which is also distance nonincreasing. Let $\Delta:=X \backslash X_{0}$.

It is proved in [2] that if $\mathscr{D}$ is normal, then $X$ is CAT( $(-1)$, and the inclusion $X_{0} \rightarrow X$ is a weak homotopy equivalence, in particular, $X_{0}$ is contractible.

Let $\Gamma$ be the group of all $p_{0}$-lifts of elements of $G$. There is a surjection $\Gamma \rightarrow G$ whose kernel is the group of automorphisms of $p_{0}$. Since $G$ acts isometrically on $Y_{0}$, the group $\Gamma$ acts isometrically on $X_{0}$, and hence on $X$, and $p$ is equivariant with respect to the surjection $\Gamma \rightarrow G$. The action of $\Gamma$ on $X_{0}$ is properly discontinuous, so since $X_{0}$ is simply-connected, $\Gamma$ can be identified with the orbifold fundamental group of $X_{0} / \Gamma=Y_{0} / G$. The sets $\Delta$ and $p^{-1}(C)$ are $\Gamma$-invariant, and $\Gamma$ permutes components of $p^{-1}(C)$.

Some difficulties in studying this $\Gamma$-action on $X$ and $p^{-1}(C)$ are due to the fact that $X$ is not locally-compact, some points have infinite stabilizers in $\Gamma$, and the set of components of $p^{-1}(C)$ is not locally finite.

\section{Preliminary results}

We keep notations and assumptions of Section 3.

Lemma 4.1. (1) $\Delta=p^{-1}(D) \subset p^{-1}(\operatorname{Int}(C))=\operatorname{Int}\left(p^{-1}(C)\right)$.

(2) $p^{-1}(\partial C)$ is the boundary of $p^{-1}(C)$.

(3) There is $r_{0}>0$ such that $r_{0}$-neighborhood of $p^{-1}(\partial C)$ is disjoint from $\Delta$, and different path-components of $p^{-1}(C)$ have disjoint $r_{0}$-neighborhoods.

(4) Path-components of $p^{-1}(C)$ coincide with connected components, and in particular are closed in $X$.

(5) If $A$ is a component of $p^{-1}(C)$, then $A$ is the closure of $A \cap X_{0}$, and the stabilizers of $A$ and of $A \cap X_{0}$ in $\Gamma$ are equal. 
Proof. (1) All the unions in

$$
X_{0} \cup p^{-1}(D)=p^{-1}\left(Y_{0}\right) \cup p^{-1}(D)=p^{-1}(Y) \subset X_{0} \cup \Delta
$$

are disjoint, so $p^{-1}(D) \subset \Delta$. Conversely, $\Delta \subset p^{-1}(D)$ else there is $x \in \Delta$ with $p(x) \in Y_{0}$, so $x \in X_{0}$ contradicting $X_{0} \cap \Delta=\emptyset$, proving the first equality. As $D \subset \operatorname{Int}(C)$, we get $p^{-1}(D) \subset p^{-1}(\operatorname{Int}(C))$, and it is trivial that $p^{-1}(\operatorname{Int}(C)) \subset$ $\operatorname{Int}\left(p^{-1}(C)\right)$. The last inclusion is an equality for if $x \in \operatorname{Int}\left(p^{-1}(C)\right)$, then either $x \in \Delta \subset p^{-1}(\operatorname{Int}(C))$, or $x \in X_{0}$, in which case a small neighborhood of $x$ lies in $X_{0} \cap p^{-1}(C)$, hence a small neighborhood of $p(x)$ lies in $Y_{0} \cap \operatorname{Int}(C)$, implying $x \in p^{-1}(\operatorname{Int}(C))$.

(2) Since $C$ is closed, so is $p^{-1}(C)$, hence the boundary of $p^{-1}(C)$ is equal to $p^{-1}(C) \backslash \operatorname{Int}\left(p^{-1}(C)\right)$; the claim now follows as all unions in

$$
p^{-1}(C)=p^{-1}(\partial C) \cup p^{-1}(\operatorname{Int}(C))=p^{-1}(\partial C) \cup \operatorname{Int}\left(p^{-1}(C)\right)
$$

are disjoint, where the second equality follows from (1).

(3) The claim follows from (2) and the fact that the submanifold $p^{-1}(\partial C) \subset X_{0}$ has positive normal injectivity radius.

(4) If a connected component contains more than one path-component, it cannot be connected by (3), so components and path-components of $p^{-1}(C)$ coincide. Since $C$ is closed, so is $p^{-1}(C)$. Components of a closed set are closed (as the closure of a connected space is connected).

(5) The closure of $A \cap X_{0}$ lies in $\bar{A}$ which equals $A$ by (4). To see that any $a \in A$ lies in the closure of $A \cap X_{0}$ it is enough to consider $a \in A \cap \Delta$, which is a limit of some sequence $x_{i} \in X_{0}$, where $x_{i} \in A$ for large $i$ as (1) implies $A \cap \Delta \subset A \cap \operatorname{Int}\left(p^{-1}(C)\right)=\operatorname{Int}(A)$.

Element of $\Gamma$ that stabilize $A$ also stabilize $A \cap X_{0}$ because $X_{0}$ is $\Gamma$-invariant, and conversely, element of $\Gamma$ that stabilize $A \cap X_{0}$, also stabilize its closure, which is $A$.

Remark 4.2. In view of (4) we refer to path-components of $p^{-1}(C)$ as components. Lemma 4.1 also implies that if $A$ is a component of $p^{-1}(C)$, then $\partial A=A \cap p^{-1}(\partial C)$.

Lemma 4.3. Let $A$ be a component of $p^{-1}(C)$. If $\mathscr{D}$ is normal, then

(i) $A$ is convex, and the orthogonal projection $X \rightarrow A$ maps $X \backslash A$ onto $\partial A$.

(ii) $A \cap X_{0}, p\left(A \cap X_{0}\right), p(A)$ are components of $p^{-1}(C) \cap X_{0}, C \cap Y_{0}, C$, respectively. The map $p: A \cap X_{0} \rightarrow p\left(A \cap X_{0}\right)$ is a universal covering, and $A \cap X_{0}$ is contractible.

Proof. (i) Path-connected locally convex subsets of CAT(0) spaces are convex ([16], Proposition II.4.14), so convexity of $A$ would follow from local convexity of $p^{-1}(C)$. Since $p: X_{0} \rightarrow Y_{0}$ is locally isometric and $C \cap Y_{0}$ is locally convex, we know that 
$p^{-1}\left(C \cap Y_{0}\right)=p^{-1}(C) \cap X_{0}$ is locally convex, so it remains to check local convexity of $p^{-1}(C)$ at the points of $p^{-1}(C) \cap \Delta$ which by Lemma 4.1 equals

$$
p^{-1}(C) \cap p^{-1}(D)=p^{-1}(C \cap D) \subset p^{-1}(\operatorname{Int}(C)) \subset \operatorname{Int}\left(p^{-1}(C)\right),
$$

but in $\operatorname{Int}\left(p^{-1}(C)\right)$ locally convexity follows from local convexity of $X$.

By Lemma 4.1(4) the subset $A$ is closed, so there is the orthogonal projection of $X$ onto $A$ that associates to a point of $X$ its (unique) nearest point in $A$. It follows that the projection maps $X \backslash A$ to $\partial A$.

(ii) It can be deduced from the proof of Lemma 3.3 in [2] that $A \backslash \Delta \rightarrow A$ is a weak homotopy equivalence. Since by (i) $A$ is convex, it is contractible, and hence so is $A \backslash \Delta=A \cap X_{0}$. In fact, $A \cap X_{0}$ is a path-component of $p^{-1}(C) \cap X_{0}$ because any path in $p^{-1}(C) \cap X_{0}$ that starts in $A \cap X_{0}$ must lie in $A$. Thus the subset $A \cap X_{0}$ is open and closed in $p^{-1}(C) \cap X_{0}$. Since $p_{0}$ is a local homeomorphism, the subset $p\left(A \cap X_{0}\right)=p(A) \cap Y_{0}$ is open and closed in $C \cap Y_{0}$, and hence $p\left(A \cap X_{0}\right)$ is a component of $C \cap Y_{0}$. Thus $p: A \cap X_{0} \rightarrow p\left(A \cap X_{0}\right)$ is a covering map of connected manifolds, which is universal as $A \cap X_{0}$ is contractible.

Finally, we show that $p(A)$ is a component of $C$. Let $Q$ be the component of $C$ that contains $p(A)$. Then $Q \cap Y_{0}$ is the component of $C \cap Y_{0}$ containing $p(A) \cap Y_{0}$, so $p(A) \cap Y_{0}=Q \cap Y_{0}$. Any $y \in Q \cap D$ is the endpoint of a geodesic segment in $Q \cap Y_{0}$. Lift the segment to the cover $A \cap X_{0}$. The lift is isometric, so along the lifted segment one gets a Cauchy sequence converging to some $x \in \Delta$. Then $y=p(x)$, and $x \in A$ because $A$ is closed. Thus $Q \cap D \subset p(A)$, which together with $Q \cap Y_{0}=p(A) \cap Y_{0}$ implies $Q \subset p(A)$, as wanted.

To state the main result of this section we let $\pi_{1}^{\text {orb }}$ denote the orbifold fundamental group.

Theorem 4.4. With notations and assumptions of Section 3 , if $\mathcal{D}$ is normal, then $\Gamma$ is non-elementary relatively hyperbolic. Under the identification $\Gamma \cong \pi_{1}^{\mathrm{orb}}\left(X_{0} / \Gamma\right) \cong$ $\pi_{1}^{\mathrm{orb}}\left(Y_{0} / G\right)$ conjugacy classes of peripheral subgroups of $\Gamma$ correspond to orbifold fundamental groups of components of $\left(C \cap Y_{0}\right) / G$, considered as subgroups of $\pi_{1}^{\text {orb }}\left(Y_{0} / G\right)$.

Remark 4.5. It is implicit in the conclusion of the above theorems that when $G$ acts freely on $Y_{0}$, the inclusion $\left(C \cap Y_{0}\right) / G \hookrightarrow Y_{0} / G$ is $\pi_{1}$-injective, and in fact it induces an isomorphism on higher homotopy groups because $\left(C \cap Y_{0}\right) / G, Y_{0} / G$ are aspherical.

Proof. We are to check that Theorem 2.1 applies to the family of components of $p^{-1}(C)$. As mentioned before, Allcock showed that $X$ is $\operatorname{CAT}(-1)$ when $\mathscr{D}$ is normal. Components of $p^{-1}(C)$ are convex by Lemma 4.3 (i), and $\varepsilon$-separated for some small positive $\varepsilon$ because $\partial C$ has positive normal injectivity radius and the tubular $\varepsilon$-neighborhood of $\partial C$ lifts to a tubular $\varepsilon$-neighborhood of $p^{-1}(\partial C)$. Lemma 4.1 (1) 
implies that the complement of $\operatorname{Int}\left(p^{-1}(C)\right)$ in $X$ is locally compact; moreover, $\Gamma$ acts on the complement properly discontinuously, and cocompactly: the former again follows from $\Delta \subset \operatorname{Int}\left(p^{-1}(C)\right)$ and the latter holds by identifying the quotient with $(Y \backslash \operatorname{Int}(C)) / G$, which is compact by assumption. Thus Theorem 2.1 implies that $\Gamma$ is hyperbolic relative to the family of stabilizers of components of $p^{-1}(C)$, which by Lemma $4.1(5)$ and Lemma 4.3 (ii) is equal to the stabilizers of components of $p^{-1}(C) \cap X_{0}$.

That $\Gamma$ acts properly discontinuously on $X_{0}$ with quotient $X_{0} / \Gamma=Y_{0} / G$, and that components of $p^{-1}(C) \cap X_{0}$ are $\varepsilon$-separated easily implies that every component of $\left(C \cap Y_{0}\right) / G$ is the quotient of some component of $p^{-1}(C) \cap X_{0}$ by its stabilizer in $\Gamma$. (Indeed, fix a component $E$ of $\left(C \cap Y_{0}\right) / G$, and pick a point in $p^{-1}(E)$. That point lies in some $A \cap X_{0}$, which is path-connected so $p\left(A \cap X_{0}\right) \subset E$, and moreover the inclusion is equality by $\varepsilon$-separation. As the $\Gamma$-action permutes components of $p^{-1}(C) \cap X_{0}$, we can identify $E$ with the quotient of $A \cap X_{0}$ by its stabilizer in $\Gamma$.)

By Lemma 4.3 (ii) components of $p^{-1}(C) \cap X_{0}$ are simply-connected, so in the above notation the stabilizer of $A \cap X_{0}$ in $\Gamma$ can be identified with the orbifold fundamental group of $E$.

To see that $\Gamma$ is non-elementary first note that $\Gamma$ is not virtually cyclic ( $\Gamma$ surjects onto $G$, and $G$ contains a non-abelian free subgroup, being a lattice in a negatively pinched Hadamard manifold). If $\Gamma$ is equal to the stabilizer of some $A$, then $G$ stabilizes $p(A)$, so $p(A)$ would have to contain the convex hull of the limit set of $G$, which is $Y$, contradicting the assumption that $C$ is a proper subset.

Remark 4.6. The above proof also implies that $G$ is non-elementary hyperbolic relative to stabilizers of components of $C$. This result is implicit in [15], and it holds regardless of whether $\mathscr{D}$ is normal by applying Theorem 2.1 to $Y$.

\section{Sparseness implies relative hyperbolicity}

We keep notations and assumptions of Section 3 except those involving $C$. For each set in $\mathscr{D} \cup \mathcal{H}$ we consider its open $\rho$-neighborhood, and let $R_{\rho}$ denote the union of these neighborhoods. Set $R_{0}:=\bigcap_{\rho>0} R_{\rho}$, i.e., $R_{0}$ is the union of all the sets in $\mathscr{D} \cup \mathcal{H}$. We suspect that $R_{\rho}$ is an open regular neighborhood of $R_{0}$, provided $\rho<\frac{r}{2}$, and this should be provable with stratified Morse theory, but for our purposes Proposition 5.1 below suffices.

Proposition 5.1. If $\mathscr{D}$ is normal, and $\{\mathscr{D}, \mathscr{H}\}$ is $r$-sparse, then for any $\rho$, $\varepsilon$ with $0<\varepsilon<\rho<\frac{r}{2}$, the inclusion $R_{\varepsilon} \cap Y_{0} \hookrightarrow R_{\rho} \cap Y_{0}$ is a homotopy equivalence.

Proof. By Whitehead's theorem it suffices to prove that the inclusion induces isomorphism on all homotopy groups. Surjectivity and injectivity will follow once we show that every compact set $K$ (such as image of sphere or disk) in $R_{\rho} \cap Y_{0}$ can be 
pushed into $R_{\varepsilon} \cap Y_{0}$ by a map that is homotopic to the identity of $R_{\rho} \cap Y_{0}$ and has the property that each point of $K \cap R_{\varepsilon} \cap Y_{0}$ stays in $R_{\varepsilon} \cap Y_{0}$ during this homotopy.

Note that $r$-sparseness imply that two arbitrary sets in $\mathcal{D} \cup \mathscr{H}$ intersect if their $\rho$-neighborhoods intersect, and normality implies that any two distinct sets in $\mathscr{D} \cup \mathscr{H}$ are disjoint or orthogonal, so that if $h, h^{\prime} \in \mathcal{D} \cup \mathscr{H}$ intersect, then the orthogonal projection $Y \rightarrow N_{\varepsilon}(h)$ takes $h^{\prime}$ to $N_{\varepsilon}(h) \cap h^{\prime}$, and also maps $N_{\rho}\left(h^{\prime}\right)$ into itself because the projection is distance non-increasing.

These properties ensure that for any subset $U$ of $N_{\rho}(h) \cap Y_{0}$, there exists a homotopy $f_{U, t}: R_{\rho} \cap Y_{0} \rightarrow R_{\rho} \cap Y_{0}$ such that

- $f_{U, 0}=$ id and $f_{U, 1}$ maps $U$ into $N_{\varepsilon}(h)$,

- tracks of $f_{U, t}$ lie on segments orthogonal to $h$,

- $f_{U, t}=$ id except possibly on tracks that pass near $U$,

- if $h \in \mathscr{D} \cup \mathscr{H}$, then $f_{U, t}$ maps $h$ and $N_{\varepsilon}(h)$ into themselves.

Now cover $K$ by finitely many precompact open sets $U_{1}, \ldots, U_{k}$ such that $U_{i}$ lies in $N_{\rho}\left(h_{i}\right) \cap Y_{0}$ where the $h_{i}$ 's are (not necessarily distinct) sets in $\mathscr{D} \cup \mathscr{H}$. Then $f_{U_{1}, t}$ pushes $U_{1}$ into $N_{\varepsilon}\left(h_{1}\right) \cap Y_{0}$. Set $U_{i}^{0}:=U_{i}$, and let $U_{i}^{1}$ be the union of tracks of $f_{U_{1}^{0}, t}$ passing through $U_{i}^{0}$; note that $U_{i}^{1}$ is precompact and $f_{U_{2}^{1}, t}$ pushes $U_{2}^{1} \supset U_{2}$ into $N_{\varepsilon}\left(h_{2}\right) \cap Y_{0}$. Let $U_{i}^{2}$ be the union of tracks of $f_{U_{2}^{1}, t}$ passing through $U_{i}^{1}$; again $U_{i}^{2}$ is precompact, and $f_{U_{3}^{2}, t}$ pushes $U_{3}^{2} \supset U_{3}$ into $N_{\varepsilon}\left(h_{3}\right) \cap Y_{0}$. Continuing in this fashion, we get homotopies $f_{U_{i}^{i-1}, t}$ pushing $U_{i}^{i-1} \supset U_{i}$ into $N_{\varepsilon}\left(h_{i}\right) \cap Y_{0}$. The composition of these homotopies pushes $K$ into $R_{\varepsilon} \cap Y_{0}$.

Theorem 5.2. There exists a constant $r>0$, depending only on $n$ and $\varkappa$, such that if $\mathcal{D}$ is normal and $\{\mathscr{D}, \mathscr{H}\}$ is $r$-sparse, then $\Gamma$ is non-elementary relatively hyperbolic. There is a positive constant $\varepsilon \ll r$ such that under the identification $\Gamma \cong \pi_{1}^{\mathrm{orb}}\left(X_{0} / \Gamma\right) \cong \pi_{1}^{\mathrm{orb}}\left(Y_{0} / G\right)$ conjugacy classes of peripheral subgroups of $\Gamma$ correspond to orbifold fundamental groups of components of $\left(R_{\varepsilon} \cap Y_{0}\right) / G$, considered as subgroups of $\pi_{1}^{\text {orb }}\left(Y_{0} / G\right)$.

Proof. To prove relative hyperbolicity the strategy is to find $C$ as in Section 3 and then apply Theorem 4.4.

Since $\{\mathscr{D}, \mathscr{H}\}$ is $r$-sparse, and sets in $\mathscr{D} \cup \mathscr{H}$ are either disjoint or orthogonal, any two points in the same component of $R_{0}$ lie on a piecewise geodesic with sidelengths $\geq r$ and angles at vertices $\geq \pi / 2$. Suppose $r>r_{1}$; then this piecewise geodesic is a $\left(\lambda_{1}, \varepsilon_{1}\right)$-quasi-geodesic, where $r_{1}, \lambda_{1}, \varepsilon_{1}$ are the constants from by Proposition A.4 corresponding to $\theta_{1}=\pi / 2$. Any subpath of a $\left(\lambda_{1}, \varepsilon_{1}\right)$-quasigeodesic, is a $\left(\lambda_{1}, \varepsilon_{1}\right)$-quasi-geodesic; thus any two points of $R_{0}$ can be joined by a $\left(\lambda_{1}, \varepsilon_{1}\right)$-quasi-geodesic. By stability of quasi-geodesics [16], Theorem III.H.1.7, there is a constant $r_{2}$ such that any $\left(\lambda_{1}, \varepsilon_{1}\right)$-quasi-geodesic in a CAT $(-1)$ space is $r_{2}$ Hausdorff close to a geodesic with the same endpoints; thus each component of $R_{0}$ is $r_{2}$-quasiconvex. Bowditch ([14], Proposition 2.5.4) proved that each $r_{2}$-quasiconvex 
subset of a Hadamard manifold with $\varkappa \leq \sec \leq-1$ must be $L=L\left(r_{2}, \varkappa\right)$-Hausdorff close to its convex hull.

For our purposes it is better to work with closed $\varepsilon$-neighborhoods of convex hulls, denoted hull $l_{\varepsilon}$, and to simplify notations we increase $L$, replacing it with $L+\varepsilon$, to ensure that any $r_{2}$-quasiconvex subset $E$ is $L$-Hausdorff close to hull $\varepsilon_{\varepsilon}(E)$.

In addition to $r>r_{1}$, suppose $r>2 L$; then distinct components of $R_{0}$ have disjoint hull $\varepsilon_{\varepsilon}$ 's because of $r$-sparseness. Let $C$ be the union of hull $\varepsilon_{\varepsilon}$ 's of the components of $R_{0}$. Thus $C$ is a $G$-invariant, closed, locally convex subset of $Y$, and furthermore, each component of $C$ is $L$-Hausdorff close to the corresponding component of $R_{0}$. As $C$ contains the $\varepsilon$-neighborhood of $D$, we have $D \subset \operatorname{Int}(C)$, and $G$ acts cocompactly on $Y \backslash \operatorname{Int}(C)$ because $C$ contains every horoball in $\mathscr{H}$. If $\varepsilon$ is sufficiently small, Remark 3.1 implies that $\partial C$ is $C^{1}$-smooth provided it is non-empty.

Lemma 5.3. There is $\alpha_{n} \in\left(0, \frac{\pi}{2}\right)$ depending only on $n=\operatorname{dim}(Y)$ such that if $2 L<r \sin \alpha_{n}$, then $C$ is a proper subset of $Y$ so that $\partial C$ is non-empty.

Proof. If $G$ acts freely, the result is immediate for homological reasons without assuming $2 L<r \sin \alpha_{n}$. Indeed, the assumption $r>2 L$ allows to apply Proposition 5.1, which yields a deformation retraction of $R_{L} / G$ onto its proper subset. Now $C \subset R_{L}$ so if $C=Y$, the deformation retraction pushes the fundamental class of $Y / G$ to a proper subset, which is impossible. This idea becomes harder to implement in the orbifold case, so we settle for an ad hoc argument below.

First we use $r$-sparseness to find $y \in Y$ such that the open ball $B\left(y, \frac{r}{2}\right)$ is disjoint from horoballs in $\mathscr{H}$, and only intersects those hyperplanes in $\mathscr{D}$ that pass through $y$.

Consider the hyperplanes $h_{1}, \ldots, h_{k}$ with nonempty intersection $h_{0}$, where we assume $k$ is the largest possible. If $y \in h_{0}$, and if $h \in \mathscr{D}$ intersects $B\left(y, \frac{r}{2}\right)$, then $h$ must intersect $h_{0}$. (Indeed, $h$ intersect each $h_{i}$ as the distance between $h, h_{i}$ is $<r$, and since $\mathscr{D}$ is normal, the orthogonal projection of $h_{i}$ onto $h$ is $h \cap h_{i}$, so the projection of $h_{0}$ lies in each $h \cap h_{i}$, and hence lies in $h \cap h_{0}$, which is therefore nonempty). Maximality of $k$ forces $h=h_{i}$ for some $i$, so $h_{1}, \ldots, h_{k}$ are the only hyperplanes in $\mathscr{D}$ that intersect $B\left(y, \frac{r}{2}\right)$. Similarly, a horoball in $\mathscr{H}$ that intersects $B\left(y, \frac{r}{2}\right)$ must intersect $h_{0}$, so if $h_{0}$ is disjoint from horoballs in $\mathscr{H}$, then any $y \in h_{0}$ has the desired property. Note that $h_{0}$ is a complete totally geodesic submanifold of $Y$, and our underlying assumptions on $\mathscr{D}, \mathscr{H}$ imply that if $h_{0}$ does intersect some $B \in \mathscr{H}$, then $h_{0}$ is asymptotic to the center of $B$. If $h_{0}$ intersects some $B \in \mathscr{H}$, then we pick $y \in h_{0}$ to be a point with $d(y, B)=\frac{r}{2}$. Then the ball $B\left(y, \frac{r}{2}\right)$ is disjoint from any horoball in $\mathscr{H}$ because distinct horoballs in $\mathscr{H}$ are $r$-separated.

A linear algebra argument shows that in the definition of a normal family of hyperplanes one can choose the linear isomorphism to be isometric with respect to the (Riemannian) inner product on the tangent space at $p$ and the Euclidean inner product on $\mathbb{R}^{n}=\mathbb{R}^{n-2 m} \times \mathbb{C}^{m}$. So there is $\alpha_{n} \in\left(0, \frac{\pi}{2}\right)$ depending only on $n=\operatorname{dim}(Y)$, and a vector $v \in T_{y} Y$ that forms angle $\geq \alpha_{n}$ with any hyperplane in $\mathscr{D}$ through $y$. Issue a geodesic in the direction of $v$, and denote by $y_{v}$ the point where it hits $\partial B\left(y, \frac{r}{2}\right)$. Let 
$z_{v}$ be a point on a hyperplane in $\mathscr{D}$ through $y$ that is closest to $y_{v}$. In the comparison triangle $\bar{\Delta}\left(\bar{y}, \bar{y}_{v}, \bar{z}_{v}\right)$ in $\mathbb{R}^{2}$ the angles at $\bar{y}, \bar{z}_{v}$ are $\geq \alpha_{n}, \geq \frac{\pi}{2}$ respectively, so the sine law yields $d\left(y_{v}, z_{v}\right)=d\left(\bar{y}_{v}, \bar{z}_{v}\right) \geq \frac{r}{2} \sin \alpha_{n}$. To finish the proof of the lemma we show that $y_{v} \notin C$. Indeed, if $y_{v}$ were in $C$, then $y_{v}$ would lie in the $L$-neighborhood of some set in $\mathscr{D} \cup \mathcal{H}$. By the above, this set would be a hyperplane through $y$, hence $L \geq d\left(y_{v}, z_{v}\right) \geq \frac{r}{2} \sin \alpha_{n}$, contradicting the assumption $L<\frac{r}{2} \sin \alpha_{n}$.

Continuing the proof of Theorem 5.2, we invoke Theorem 4.4 to conclude that $\Gamma$ is non-elementary relatively hyperbolic.

It remains to identify orbifold fundamental groups of corresponding components of $\left(R_{\varepsilon} \cap Y_{0}\right) / G$ and $\left(C \cap Y_{0}\right) / G$. The construction of $C$ implies $R_{0} \subset R_{\varepsilon} \subset C \subset R_{L}$. By Proposition 5.1 the inclusion $R_{\varepsilon} \cap Y_{0} \rightarrow R_{L} \cap Y_{0}$ is a homotopy equivalence that factors through $C \cap Y_{0}$. So the inclusion $C \cap Y_{0} \rightarrow R_{L} \cap Y_{0}$ is surjective on homotopy groups, and moreover is injective on homotopy groups for if $f: S^{k} \rightarrow C \cap Y_{0}$ is null-homotopic in $R_{L} \cap Y_{0}$, then this homotopy can be pushed to $C$ by composing it with the orthogonal projection of $Y$ onto the component of $C$ that contains $f\left(S^{k}\right)$. Thus the inclusion $R_{\varepsilon} \cap Y_{0} \rightarrow C \cap Y_{0}$ is a homotopy equivalence.

Fix an arbitrary component $K$ of $C$, and set $K_{\varepsilon}:=K \cap R_{\varepsilon}$ and $K_{0}:=K \cap R_{0}$. Note that $\operatorname{Stab}_{G}\left(K \cap Y_{0}\right)=\operatorname{Stab}_{G}\left(K_{\varepsilon} \cap Y_{0}\right)$, i.e. $K \cap Y_{0}$ and $K_{\varepsilon} \cap Y_{0}$ have equal stabilizers in $G$. Indeed, $K$ is $\operatorname{Stab}_{G}\left(K \cap Y_{0}\right)$-invariant, and therefore so is $K_{0}$, which implies that $\operatorname{Stab}_{G}\left(K \cap Y_{0}\right)$ preserves $K_{\varepsilon}$, and hence $K_{\varepsilon} \cap Y_{0}$. On the other hand, $\operatorname{Stab}_{G}\left(K_{\varepsilon} \cap Y_{0}\right)$ preserves $K_{\varepsilon}$, and hence $K_{0}$, which means that it preserves $K=\operatorname{hull}_{\varepsilon}\left(K_{0}\right)$, and hence $K \cap Y_{0}$.

Since the inclusion $R_{\varepsilon} \cap Y_{0} \rightarrow C \cap Y_{0}$ is a homotopy equivalence, so is the inclusion $K_{\varepsilon} \cap Y_{0} \hookrightarrow K \cap Y_{0}$, hence it lifts to a homotopy equivalence of universal covers $\widetilde{K_{\varepsilon} \cap Y_{0}} \hookrightarrow \widetilde{K \cap Y_{0}}$. In particular, the preimage of $K_{\varepsilon} \cap Y_{0}$ under the universal cover $\widetilde{K \cap Y_{0}} \rightarrow K \cap Y_{0}$ is connected, and we identify it with $\widetilde{K_{\varepsilon} \cap Y_{0}}$. Also Lemma 4.3 allows us identify the universal cover $\widetilde{K \cap Y_{0}} \rightarrow K \cap Y_{0}$ with the restriction of $p$ to a component of $p^{-1}(C) \cap X_{0}$. With these identifications it follows that $\operatorname{Stab}_{\Gamma}\left(\widetilde{K \cap Y_{0}}\right)=\operatorname{Stab}_{\Gamma}\left(\widetilde{K_{\varepsilon} \cap Y_{0}}\right)$, which is exactly what we claimed (translating to orbifold terminology).

Addendum 5.4. Under the assumptions of Theorem 5.2, if $G$ acts freely on $Y$, then each component of $\left(C \cap Y_{0}\right) / G$ is aspherical, and both inclusions

$$
\left(R_{\varepsilon} \backslash R_{0}\right) / G \hookrightarrow\left(R_{\varepsilon} \cap Y_{0}\right) / G \hookrightarrow\left(C \cap Y_{0}\right) / G
$$

are homotopy equivalences.

Proof. Contracting along radial geodesics in each horoball in $\mathscr{H}$ is a $G$-equivariant deformation retraction $R_{\rho} \cap Y_{0} \rightarrow R_{\rho} \backslash R_{0}$, which descends to a homotopy equivalence $\left(R_{\varepsilon} \backslash R_{0}\right) / G \hookrightarrow\left(R_{\varepsilon} \cap Y_{0}\right) / G$. Checking that the other inclusion is a homotopy equivalence is best done one component at a time, so let $K$ denote a component of 
$C$ and use associated notations from the proof of Theorem 5.2. By Lemma 4.3 (ii) $\widetilde{K \cap Y_{0}}$ is contractible, so $K_{\varepsilon} \cap Y_{0}, K \cap Y_{0}$ are aspherical. The last paragraph in the proof of Theorem 5.2 implies that the inclusion

$$
\left(K_{\varepsilon} \cap Y_{0}\right) / \operatorname{Stab}_{G}\left(K \cap Y_{0}\right) \hookrightarrow\left(K \cap Y_{0}\right) / \operatorname{Stab}_{G}\left(K \cap Y_{0}\right)
$$

induces a $\pi_{1}$-isomorphism of aspherical manifolds, and hence a homotopy equivalence.

Proof of Theorem 1.4. To simplify notations set $Z:=R_{0} / G$; this was also denoted $Q \cup S$ in the introduction. Fix a smooth closed regular neighborhood $T$ of $Z$ inside $\left(C \cap Y_{0}\right) / G$, and show that the inclusion $\partial T \hookrightarrow\left(C \cap Y_{0}\right) / G$ is a homotopy equivalence. To this end pick $\varepsilon$ small enough such that $R_{\varepsilon} / G$ lies inside $T$, and let $T_{\varepsilon}$ denote a regular neighborhood of $Z$ inside $R_{\varepsilon} / G$. Then we have inclusions

$$
T_{\varepsilon} \backslash Z \underset{i}{\hookrightarrow}\left(R_{\varepsilon} \backslash R_{0}\right) / G \underset{j}{\hookrightarrow} T \backslash Z \underset{k}{\hookrightarrow}\left(C \cap Y_{0}\right) / G
$$

Standard properties of regular neighborhoods (see e.g. [17]) imply that $j \circ i$ is a homotopy equivalence, as $T \backslash Z$ is the union along $\partial T_{\varepsilon}$ of $T \backslash \operatorname{Int}\left(T_{\varepsilon}\right)$ and $T_{\varepsilon} \backslash Z$, which are diffeomorphic to $\partial T_{\varepsilon} \times[0,1]$ and $\partial T_{\varepsilon} \times[0,1)$, respectively. Addendum 5.4 says that $k \circ j$ is a homotopy equivalence. This easily implies that $j$ induces isomorphism on homotopy groups, hence $i, j, k$ are also homotopy equivalences. Finally, $\partial T \hookrightarrow$ $T \backslash Z \cong \partial T \times[0,1)$ is a homotopy equivalence, and hence so is the inclusion $\partial T \hookrightarrow$ $\left(C \cap Y_{0}\right) / G$, thus components of $\partial T$ are aspherical and $\pi_{1}$-injectively embedded, proving (1)-(2). The assertions (3)-(4) are immediate from Theorem 5.2.

Parts (5) and (7) follow from Theorem 1.3 of [7] saying that for any compact aspherical manifold $N$ that satisfies (1)-(4), the group $\pi_{1}(N)$ is co-Hopf, and $\pi_{1}(N)$ does not split as an amalgamated product or HNN-extension over subgroups that are elementary in the relatively hyperbolic group structure given by (3)-(4).

To prove (6) recall that Drutu and Sapir ([23], Theorem 1.12) showed that if none of the peripheral subgroups of a relatively hyperbolic group $H$ is isomorphic to a non-elementary relatively hyperbolic group, and if $H$ does not split over an elementary subgroup, then $\operatorname{Out}(H)$ is finite. By the previous paragraph $\pi_{1}(N)$ does not split over elementary subgroups. Components of $\partial N$ are closed aspherical manifolds, so by Proposition 1.6 if any of them has non-elementary relatively hyperbolic fundamental group, then that component has positive simplicial volume, which we assume arguing by contradiction. Since $S$ is compact, the assumptions in Section 3 imply that $S$ and $Q$ are disjoint, so components of $\partial N$ are either components of $\partial Q$, or the boundary components of a small regular neighborhood of $S$. Components of $\partial Q$ are infranilmanifolds, so they have zero simplicial volume. Thus the boundary of a regular neighborhood of $S$ has positive simplicial volume. Let $D M$ be the manifold obtained by doubling $M \backslash \operatorname{Int}(Q)$ along the boundary, which is $\partial Q$; we think of $S$ as sitting in one half of the double. Then $D M \backslash S$ must have infinite simplicial volume [27], page 17 (cf. [27], pages 56-57), as the interior of a compact manifold 
whose boundary has positive simplicial volume. This gives a contradiction as $D M \backslash S$ has finite simplicial volume by [27], page 59, with details given in Lemma 5.6 below. Thus we have proved (6).

For part (8) recall that each peripheral subgroup of $\pi_{1}(N)$ is the fundamental group of a closed aspherical 3-manifold, which are geometrizable due to work of Thurston, Perelman and others. (To our knowledge the Ricci flow proof of the geometrization conjecture has been fully written only for orientable manifolds, but non-orientable aspherical 3-manifolds are Haken [31], so Thurston's proof applies in this case. Actually, our argument below can be phrased to depend only on the geometrization of orientable manifolds but we felt it would only confuse the matters.)

Hempel [30] proved that geometrizable 3-manifold groups are residually finite, and in particular, they have solvable word problem. Preaux [38], [39] showed that fundamental groups of 3-manifold with geometrizable orientation covers have solvable conjugacy problem. According to Farb [25] and Bumagin [18], a relatively hyperbolic group inherits solvability of word and conjugacy problems from its peripheral subgroups, hence these problems are solvable for $\pi_{1}(N)$. As in the proof of Theorem 1.1 (5) of [9], the result of Osin [35] and residual finiteness of the peripheral subgroups implies that $\pi_{1}(N)$ is (fully) residually hyperbolic.

By the proof of (6), finiteness of $\operatorname{Out}\left(\pi_{1}(N)\right)$ would follow if we find a relatively hyperbolic group structure on $\pi_{1}(N)$ such that every peripheral subgroup is elementary in the relatively hyperbolic group structure given by (3)-(4), and also is not isomorphic to a non-elementary relatively hyperbolic group.

According to Corollary 1.14 of [22], if $H$ is hyperbolic relative to $\left\{P_{i}\right\}$ and if each $P_{i}$ is hyperbolic relative to $\left\{P_{i}^{j}\right\}$, where we allow $P_{i}$ to equal $P_{i}^{j}$, then $H$ is hyperbolic relative to the $\left\{P_{i}^{j}\right\}$ 's. This process can be iterated, and in general need not terminate, but as we note below it does terminate if we start with the fundamental group of a closed aspherical 3-manifold, which would complete the proof that $\operatorname{Out}\left(\pi_{1}(N)\right)$ is finite. Fix a closed aspherical 3-manifold $M$. By the geometrization theorem, $M$ is hyperbolic, Sol, Seifert fibered, or has a nontrivial JSJ decomposition along incompressible tori and Klein bottles whose pieces are either hyperbolic or Seifert fibered. Recall that the simplicial volume $\|M\|$ is nonzero if and only if $M$ is either hyperbolic or has a hyperbolic piece in the JSJ decomposition. If $\|M\|=0$, then Proposition 1.6 implies that $\pi_{1}(M)$ is not non-elementary relatively hyperbolic, so the process terminates with $\pi_{1}(M)$. If $M$ is hyperbolic, the process terminates with the trivial subgroup. It remains to consider the case when there is a hyperbolic piece $\mathscr{H}$ in the JSJ decomposition. Then $\pi_{1}(M)$ is hyperbolic relative to fundamental groups of the components of $M \backslash \mathscr{H}$, as follows e.g. from Dahmani's combination theorem [21] and the fact that $\pi_{1}(\mathscr{H})$ is hyperbolic relative to fundamental groups of tori and Klein bottles that lie on $\partial \mathscr{H}$. So passing to the peripheral subgroups corresponding to the components of $M \backslash \mathscr{H}$, and continuing in this fashion, after finitely many steps we end up with components that are either aspherical graph manifolds with incompressible boundary, or surfaces of zero Euler characteristic appearing on the 
boundary of hyperbolic pieces. In either case the process terminates; indeed, by Lemma 5.5 below, or alternatively by Theorem 11.1 of [6], the fundamental group of an aspherical graph manifold with incompressible boundary is not non-elementary relatively hyperbolic, and the same holds for surfaces of zero Euler characteristic because any non-elementary relatively hyperbolic group contains $\mathbb{Z} * \mathbb{Z}$, so it is not virtually- $\mathbb{Z}^{2}$.

Osin [34] proved that a relatively hyperbolic group has finite asymptotic dimension if so do all the peripheral subgroups. Bell-Dranishnikov[10], [11] showed that the class of finitely generated groups of finite asymptotic dimension is closed under extensions, amalgamated products, and $\mathrm{HNN}$-extensions. By the previous paragraph, one can build $\pi_{1}(N)$ in finitely many steps starting from the trivial group and using extensions, amalgamated products, HNN-extensions, and passing to peripheral subgroups, so that $\pi_{1}(N)$ has finite asymptotic dimension. This completes the proof of (8), and hence of Theorem 1.4.

Lemma 5.5. If $M$ is a compact aspherical graph manifold such that $\partial M$ is incompressible and has zero Euler characteristic, then $\pi_{1}(M)$ is not isomorphic to a non-elementary relatively hyperbolic group.

Proof. Arguing by contradiction suppose that $\pi_{1}(M)$ is non-elementary relatively hyperbolic. Then the fundamental group of each component of $\partial M$ must lie in a peripheral subgroup, like all virtually- $\mathbb{Z}^{2}$ subgroups do. Let $D M$ denote the double of $M$ along $\partial M$. By Dahmani's combination theorem [21] the relatively hyperbolic group structure on $\pi_{1}(M)$ defines a non-elementary relatively hyperbolic structure on $\pi_{1}(D M)$. Then Proposition 1.6 implies $\|D M\|>0$, which is false as graph manifolds have zero simplicial volume.

Lemma 5.6. If $S$ is normal and compact, then $\|D M \backslash S\|$ is finite.

Proof. A proof of finiteness of $\|D M \backslash S\|$ is sketched in [27], pages 58-59; we fill the details with the help of [32], Theorem 5.3. To state Gromov's result we need two definitions. A subset of a space is called amenable if for any choice of the basepoint the $\pi_{1}$-homomorphism induced by inclusion has amenable image. A sequence of subsets $U_{i}$ of a space $X$ is called amenable at infinity if there is an exhaustion of $X$ by a sequence of compact sets $K_{i}$ such that $K_{i} \subset K_{i+1}, U_{i} \subset X \backslash K_{i}$, and $U_{i}$ is amenable in $X \backslash K_{i}$ for large $i$.

A special case of Gromov's Finiteness Theorem ([27], bottom of page 58) says the following: an $n$-dimensional manifold $V$ has finite simplicial volume if $V$ admits a locally finite cover by precompact open sets $U_{i}$ that are amenable at infinity, and such that the cover $\left\{U_{i}\right\}$ has multiplicity $\leq n$ over a subset that has precompact complement in $V$.

We extend the metric on $M \backslash Q$ arbitrarily to a metric on $D M$. Since $S$ is compact and normal in $D M$, we can cover $S$ by finitely many small metric balls with centers 
in $S$ such that for each such ball $B$ we have $\pi_{1}(B \backslash S)$ is free abelian. Since $S$ is $(n-2)$-dimensional, this cover has a finite refinement $\left\{B_{j}\right\}$ of multiplicity $\leq n-1$, and we index this cover by a finite set $J$. Choose the balls sufficiently small so that the original cover lies in some (closed) regular neighborhood $T_{0}$ of $S$ in $D M$. Also choose a sequence of regular neighborhoods $T_{k}$ of $S$ all covered by $\left\{B_{j}\right\}$ with $\bigcap_{k} T_{k}=S$ and $T_{k} \supset T_{k+1}$.

Fix a proper function $f: T_{0} \backslash S \rightarrow \mathbb{R}$. As in the proof of Theorem 5.3 of [32] for each $j \in J$ we find a cover $U_{j}$ of $\mathbb{R}$ by bounded open intervals of multiplicity 2 such that $U_{i} \cup U_{j}$ has multiplicity 3 if $i \neq j$; this uses finiteness of $J$. Consider the cover of $T_{0} \backslash S$ by sets $B_{j} \cap f^{-1}(U)$ with $U \in \mathcal{U}_{j}$. Each $B_{j} \cap f^{-1}(U)$ is relatively compact because sets in $U_{j}$ are bounded and $f$ is proper. Thus we have covered $T_{1} \backslash S$ by relatively compact open subsets $B_{j} \cap f^{-1}(U)$. By an elementary argument in the proof of Theorem 5.3 of [32] this cover has multiplicity $\leq n$. It remains to show this cover is amenable at infinity, i.e., if $B_{j} \cup f^{-1}(U)$ lies in some $T_{k}$, then it is amenable there. Since $T_{k} \hookrightarrow T_{0}$ is a homotopy equivalence, it is enough to show that $B_{j} \cup f^{-1}(U)$ is amenable in $T_{0}$, which follows as the inclusion factors through the abelian group $\pi_{1}\left(B_{j} \backslash S\right)$.

Remark 5.7. An alternative way to prove that the boundary of a regular neighborhood of $S$ has zero simplicial volume would be to show that it admits an $F$-structure, and then apply the result of Paternain-Petean [36] that any manifold with an $F$-structure has zero simplicial volume. The existence of an $F$-structure, or even the existence of local torus actions that commute on overlaps, should follow from normality of $S$, but we do not attempt proving it here, as we do not see any applications.

\section{Other applications}

In this section we prove Corollary 1.3, Proposition 1.6, Theorem 1.5, and Theorem 1.7.

Proof of Corollary 1.3. The peripheral subgroups in this case are either virtually nilpotent, or have a normal infinite cyclic subgroups with hyperbolic quotient. That $\pi_{1}(M \backslash S)$ are residually hyperbolic is provided exactly as in the proof of Corollary 1.4 (4) of [8]. That $\pi_{1}(M \backslash S)$ is co-Hopf and has finite outer automorphism group follows from Theorem 1.3 of [7], which also implies that $\pi_{1}(M \backslash S)$ does not split nontrivially over elementary subgroups. By [41] every non-elementary subgroup contains a non-abelian free subgroup. All the other asserted properties are proved exactly as in the proof of Theorem 1.1 of [9].

Proof of Proposition 1.6. We can assume that $M$ is orientable as relative hyperbolicity is inherited by finite index subgroups. Let $X$ be an Eilenberg-MacLane space for $\pi_{1}(L)$ in which peripheral subgroups are realized by $\pi_{1}$-injective inclusions of aspherical subspaces $A_{i}$, one for each conjugacy class of peripheral subgroups. Let 
$A=\bigcup_{i} A_{i}$. Peripheral subgroups of a non-elementary relatively hyperbolic group have infinite index, hence each $A_{i}$ is homotopy equivalent to an open $n$-manifold; hence $H_{n}\left(A_{i}\right)=0$, which implies $H_{n}(A)=0$. By the homology long exact sequence of the pair, the map $H_{n}(X) \rightarrow H_{n}(X, A)$ is injective, i.e., the fundamental class $[L]$ is mapped to a nonzero element. By the main result of Mineyev-Yaman [33] that element has positive simplicial norm, and since simplicial norm is non-increasing under continuous maps we conclude $\|L\|>0$.

Theorem 1.5 is the manifold version of Theorem 6.1 below, for which we adopt notations and assumptions of Section 3 except those involving $C$.

\section{Theorem 6.1. Suppose that $n>3$ and $\mathscr{D}$ is normal.}

(a) If $h \in \mathscr{D}$, then $h / \mathrm{Stab}_{G}(h)$ has finite volume.

(b) There is a nontrivial element of $\Gamma$ whose centralizer contains a non-abelian free subgroup. In particular, $\Gamma$ is not isomorphic to a discrete group of isometries of a negatively pinched Hadamard manifold.

Proof. (a) Note that $h$ is a negatively pinched manifold of dimension $\geq 2$. By Section 3, we can always arrange that if $B \in \mathcal{H}$, then either $h$ and $B$ are disjoint, or $h$ is asymptotic to the center of $B$. In the latter case $h \cap B$ is a horoball in $h$. The set $\mathscr{H}_{h}$ of horoballs in $\mathscr{H}$ that intersect $h$ is $\operatorname{Stab}_{G}(h)$-invariant. Let $Y^{c}, h^{c}$ be the complements in $Y, h$ of interiors of the horoballs in $\mathscr{H}, \mathscr{H}_{h}$, respectively. Since $Y^{c} / G$ is compact, local finiteness of $\mathscr{D}$ implies that $h^{c} / \operatorname{Stab}_{G}(h)$ is a compact subset of $Y^{c} / G$. (Otherwise, there is a sequence of points in $h^{c}$, whose projections in $h^{c} / \operatorname{Stab}_{G}(h)$ converge to a point outside $h^{c} / \operatorname{Stab}_{G}(h)$, and hence the points have lifts lying on $G$-images of $h$ and converging to a point of $Y$, contradicting local finiteness). Thus for each $B \in \mathscr{H}_{h}$, the subgroup of $\operatorname{Stab}_{G}(h)$ that preserves the horoball $h \cap B$ acts cocompactly in $\partial B \cap h$, so $h / \operatorname{Stab}_{G}(h)$ has finite volume.

(b) First suppose that there are $h, h_{1} \in \mathscr{D}$ that intersect. Since $h / \operatorname{Stab}_{G}(h)$ has finite volume, there is $g \in \operatorname{Stab}_{G}(h)$ that moves $h \cap h_{1}$ to some disjoint hyperplane in $h$, e.g., let $g$ be a hyperbolic element whose attracting limit point is not at infinity of $h \cap h_{1}$, so that powers of $g$ bring $h \cap h_{1}$ within an arbitrary small neighborhood of the attracting point. Since $h_{1}$ is orthogonal to $h$, so is $h_{2}:=g\left(h_{1}\right)$, and then $h_{1}, h_{2}$ must be disjoint. Since $\mathscr{D}$ is normal, $\pi_{1}\left(Y \backslash\left\{h, h_{1}, h_{2}\right\}\right)$ is the amalgamated product of $\pi_{1}\left(Y \backslash\left\{h, h_{1}\right\}\right) \cong \mathbb{Z}^{2}$ and $\pi_{1}\left(Y \backslash\left\{h, h_{2}\right\}\right) \cong \mathbb{Z}^{2}$ along $\pi_{1}(Y \backslash\{h\}) \cong \mathbb{Z}$. The obvious surjection $\pi_{1}(Y \backslash D) \rightarrow \pi_{1}\left(Y \backslash\left\{h, h_{1}, h_{2}\right\}\right)$ splits. (Indeed, take a smooth path in $h$ that joins $h$ to $h_{1}$ and intersects no other hyperplane in $D$. The path has a small neighborhood $V$ that intersects no hyperplane in $\mathscr{D} \backslash\left\{h, h_{1}, h_{2}\right\}$, and has the property that the inclusion-induced map $\pi_{1}\left(V \backslash\left\{h, h_{1}, h_{2}\right\}\right) \rightarrow \pi_{1}\left(Y \backslash\left\{h, h_{1}, h_{2}\right\}\right)$ is an isomorphism, hence the inclusion-induced map $\pi_{1}\left(V \backslash\left\{h, h_{1}, h_{2}\right\}\right) \rightarrow \pi_{1}(Y \backslash \mathscr{D})$ gives rise to a splitting.)) Since $\pi_{1}(Y \backslash D)$ is a subgroup of $\Gamma$, so is $\pi_{1}\left(Y \backslash\left\{h, h_{1}, h_{2}\right\}\right)$, which by the above is isomorphic to $\mathbb{Z} \times(\mathbb{Z} * \mathbb{Z})$, and hence the centralizer of an element of $\Gamma$ contains $\mathbb{Z} * \mathbb{Z}$. 
It remains to study the case when no two hyperplanes in $\mathscr{D}$ intersect. Fix any $h \in D$ D. Then $\operatorname{Stab}_{G}(h)$ acts on $Y \backslash h$, hence an index two subgroup of $\operatorname{Stab}_{G}(h)$ acts trivially on $\pi_{1}(Y \backslash h) \cong \mathbb{Z}$. By (a), $h / \operatorname{Stab}_{G}(h)$ has finite volume, so some $g_{1}, g_{2} \in \operatorname{Stab}_{G}(h)$ generate a free subgroup. Consider $\gamma \in \pi_{1}(Y \backslash D) \subset \Gamma$ generated by a loop around $h$. The loop is preserved by $g_{i}$ up to free homotopy, hence there is a lift $\gamma_{i} \in \Gamma$ of $g_{i}$ that commutes with $\gamma$. Since $g_{1}, g_{2}$ generate a free subgroup, so do $\gamma_{1}, \gamma_{2}$, as promised.

Finally, in a discrete group of isometries of a negatively pinched Hadamard manifold the centralizer $C(g)$ of any infinite order element $g$ is virtually nilpotent. (Indeed, $C(g)$ preserves the limit set of $g$, which consists of one or two points. In the former case, $C(g)$ is virtually nilpotent by [13], and in the latter case $C(g)$ is virtually$\mathbb{Z}$ because it acts properly discontinuously on the geodesic line joining the limit points).

Remark 6.2. Part (a) supplies an elementary proof for a claim made in [3] and proved later in [4].

The proposition below is immediate from the main result of [33].

Proposition 6.3. For $n>1$, let $N$ be a compact aspherical n-manifold with boundary. Then $\|N, \partial N\|>0$ if $N$ contains a codimension zero compact submanifold $U$ with $\partial N \subset \operatorname{Int}(U)$ and such that

- every component $U_{i}$ of $U$ is aspherical and $\pi_{1}$-incompressible in $N$,

- $\pi_{1}(N)$ is non-elementary hyperbolic relative to $\left\{\pi_{1}\left(U_{i}\right)\right\}$.

Proof. Suppose first that $N$ is orientable. The submanifold $L:=N \backslash \operatorname{Int}(U)$ is compact, so the fundamental class of $[L, \partial L]$ generates $H_{n}(L, \partial L) \cong \mathbb{Z}$ which equals $H_{n}(N, U)$ by excision. But in $H_{n}(N, U)$ the classes [N, $\left.\partial N\right],[L, \partial L]$ are equal, so $[N, \partial N]$ is nonzero in $H_{n}(N, U)$. By [33] nonzero classes in $H_{k}(N, U), k>1$, have positive simplicial norm; this applies to $[N, \partial N] \in H_{k}(N, U)$. Since simplicial norm is nonincreasing under continuous maps, $[N, \partial N]$ has positive simplicial norm in $H_{n}(N, \partial N)$, as claimed. The case when $N$ is non-orientable follows by working in the oriented 2 -fold covers because codimension zero embeddings of manifold are orientation-true, and relative hyperbolicity is inherited by finite index subgroups.

Proof of Theorem 1.7. Fix $Q \subset \operatorname{Int}(V)$ as in the introduction, and pick closed regular neighborhoods $T_{1}, T_{2}$ of $S \cup Q$ in $M$ so small that

$$
T_{1} \subset \operatorname{Int}\left(T_{2}\right) \subset \operatorname{Int}(V) .
$$

Proposition 6.3 applies to $N:=M \backslash \operatorname{Int}\left(T_{1}\right)$ and $U:=V \backslash \operatorname{Int}\left(T_{2}\right)$, therefore $\|N, \partial N\|>0$. It is immediate from the definitions that $\|\operatorname{Int}(N)\| \geq\|N, d N\|$, see [27], pages 17, 58, and the claimed result follows as $\operatorname{Int}(N)$ is diffeomorphic to $M \backslash S$. 


\section{A. Some facts about CAT(-1) spaces}

The results in this appendix are well known but we could not find references.

Lemma A.1. If $A, B$ are closed convex subsets of a complete CAT(-1) space $X$ such that $D:=\operatorname{dist}(A, B)>0$, then there is a unique geodesic segment with endpoints in $A, B$ of length $D$.

Proof. Let $b: X \rightarrow B$ denote the orthogonal projection. To prove existence, take $x_{i} \in A$ with $\operatorname{dist}\left(x_{i}, B\right) \in\left[D, D+\frac{1}{i}\right)$, look at the triangles $\Delta\left(x_{i}, x_{j}, b\left(x_{i}\right)\right)$, $\Delta\left(x_{j}, b\left(x_{j}\right), b\left(x_{i}\right)\right)$, and lay the corresponding comparison triangles $\Delta\left(\bar{x}_{i}, \bar{x}_{j}, \overline{b\left(x_{i}\right)}\right)$, $\Delta\left(\overline{b\left(x_{j}\right)}, \bar{x}_{j}, \overline{b\left(x_{i}\right)}\right)$ in the hyperbolic plane $\mathbf{H}^{2}$ next to each other along the side $\left[\bar{x}_{j}, \overline{b\left(x_{i}\right)}\right]$. The distance between the sides $\left[\bar{x}_{i}, \bar{x}_{j}\right],\left[\overline{b\left(x_{i}\right)}, \overline{b\left(x_{j}\right)}\right]$ is $\geq D$ for if they could be joined by a segment of length $<D$, then the parts of the segment lying on different sides of $\left[\bar{x}_{j}, \overline{b\left(x_{i}\right)}\right]$ would define, by comparison, an even shorter path concatenated from two geodesic segments and joining $\left[x_{i}, x_{j}\right] \subset A$ with $\left[b\left(x_{i}\right), b\left(x_{j}\right)\right] \subset B$. Therefore $\left[\bar{x}_{i}, \bar{x}_{j}\right]$ lies outside the $D$-neighborhood of $\left[\overline{b\left(x_{i}\right)}, \overline{b\left(x_{j}\right)}\right]$, and also inside the $D+\frac{1}{i}$-neighborhood of $\left[\overline{b\left(x_{i}\right)}, \overline{b\left(x_{j}\right)}\right]$ because the neighborhood is convex and contains the endpoints of $\left[\bar{x}_{i}, \bar{x}_{j}\right]$.

We now prove that $\left\{x_{i}\right\}$ is a Cauchy sequence. Arguing by contradiction assume that $d\left(x_{i}, x_{j(i)}\right)$ is bounded away from zero for some subsequence $j=j(i)$. Applying isometries of $\mathbf{H}^{2}$ we may assume that $\overline{b\left(x_{i}\right)}$ is independent of $i$, so as $i \rightarrow \infty$ the segments $\left[\overline{b\left(x_{i}\right)}, \overline{b\left(x_{j(i)}\right)}\right]$ subconverge to a segment $S$ (possibly of zero or infinite length), and hence $\left[\bar{x}_{i}, \bar{x}_{j(i)}\right]$ subconverge to a geodesic segment on the boundary of the $D$-neighborhood of $S$, which by elementary hyperbolic geometry contains no positive length geodesic segments. Thus the limit of $\left[\bar{x}_{i}, \bar{x}_{j(i)}\right]$ on the boundary of the $D$-neighborhood of $S$ is a point, and hence $d\left(x_{i}, x_{j(i)}\right)=d\left(\bar{x}_{i}, \bar{x}_{j(i)}\right) \rightarrow 0$.

Since $X$ is complete and $A$ is closed, $\left\{x_{i}\right\}$ has a limit $x \in A$, and $[x, b(x)]$ is a segment of length $D$ joining $A, B$. Uniqueness also follows for if $[x, b(x)]$ and $[y, b(y)]$ are length $D$ segments joining $A$ and $B$, then $[\bar{x}, \bar{y}]$ lies on the boundary of the $D$-neighborhood of $[\overline{b(x)}, \overline{b(y)}]$, forcing $\bar{x}=\bar{y}$ and hence $x=y$.

Remark A.2. Existence of a shortest geodesic joining $A, B$ fails if $D=0$ (for asymptotic geodesics in $\mathbf{H}^{2}$ ), or if $D>0$ but $X=\mathbb{R}^{2}$ (for the subsets $y \geq e^{x}$ and $y \leq-1)$.

We refer to Section 2 for the definition of $r$-separation and bounded penetration.

Lemma A.3. Any family of $r$-separated convex subspaces of a CAT(-1) space has bounded penetration.

Proof. Otherwise there is $D$ such that for each $i$ there are $r$-separated convex subsets $C_{i}, B_{i}$ in the family and points $c_{i}, c_{i}^{\prime} \in C_{i}, b_{i}, b_{i}^{\prime} \in B_{i}$ such that $d\left(c_{i}, b_{i}\right)+d\left(c_{i}^{\prime}, b_{i}^{\prime}\right)<$ 
$D$, while $d\left(c_{i}, c_{i}^{\prime}\right)>i, d\left(b_{i}, b_{i}^{\prime}\right)>i$. By comparison with the hyperbolic plane there is $\varepsilon_{i} \rightarrow 0$ such that the midpoint of $\left[c_{i}, c_{i}^{\prime}\right] \subset C_{i}$ is $\varepsilon_{i}$-close to a point of $\left[c_{i}, b_{i}^{\prime}\right]$ which in turn is $\varepsilon_{i}$-close to a point of $\left[b_{i}, b_{i}^{\prime}\right] \subset B$, and we get a contradiction for $i$ with $2 \varepsilon_{i}<r$.

The following generalizes Lemma 11.3.4 of [24] on piecewise geodesics in the real hyperbolic space.

Proposition A.4. For each angle $\theta_{1}>0$ there are constants $r_{1}, \lambda_{1}, \varepsilon_{1}$ such that any piecewise geodesic in a CAT (-1) space whose geodesic pieces have length $\geq r_{1}$ and such that successive pieces meet at an angle $\geq \theta_{1}$ is a $\left(\lambda_{1}, \varepsilon_{1}\right)$-quasi-geodesic.

Proof. We are to apply Theorem 3.1.4 of [20] that for any constants $\delta, \lambda_{0}, \epsilon_{0}$ there are constants $\lambda_{1}, \epsilon_{1}, r_{1}$ such any $r_{1}$-local $\left(\lambda_{0}, \epsilon_{0}\right)$-quasi-geodesic in a $\delta$-hyperbolic space is a global $\left(\lambda_{1}, \varepsilon_{1}\right)$-quasi-geodesic.

Fix $\delta$ such that any CAT( $(-1)$ space $\delta$-hyperbolic [16], Proposition III.H.1.2. Let $c_{1}, c_{2}$ be two geodesic rays in the hyperbolic plane emanating from a common point $p$ at angle $\theta$. By an elementary computation $f(\theta, t):=d\left(c_{1}(t), c_{2}(t)\right)$ is increasing in $\theta$ for each fixed $t>0$, so let $\rho=\rho\left(\theta_{1}\right)$ be the unique solution of $f\left(\theta_{1}, \rho\right)=2 \delta$. Then let $\lambda_{0}:=1$ and $\varepsilon_{0}:=2 \delta+2 \rho$, and let $\lambda_{1}, \varepsilon_{1}, r_{1}$ be the constants given by the above-mentioned Theorem 3.1.4 in [20].

Given a piecewise geodesic $c$ whose pieces have length $\geq r_{1}$ and meet at angles $\geq \theta_{1}$, it remains to verify that $c$ is an $r_{1}$-local $\left(1, \varepsilon_{0}\right)$-quasi-geodesic, i.e., for any points $p, q$ on the quasi-geodesic with $d(p, q) \leq r_{1}$ we need to check that the distance between $p, q$ along $c$ is $\leq d(p, q)+\varepsilon_{0}=d(p, q)+2 \delta+2 \rho$. It is enough to consider $p, q$ lying on consecutive geodesic pieces of $c$; suppose they form the angle $\theta$ at their common vertex $u$.

If either $p$ or $q$, say $q$, is within distance $\rho$ of $u$, then

$$
d(p, q) \geq d(p, u)-d(u, q) \geq d(p, u)+d(u, q)-2 \rho,
$$

as desired, so assume that both $d(p, u)$ and $d(q, u)$ are $\geq \rho$. Consider the geodesic triangle $\Delta=\Delta(p, q, u)$ and a comparison triangle $\bar{\Delta}=\overline{\bar{\Delta}}(\bar{p}, \bar{q}, \bar{u})$ in the hyperbolic plane. Take $a \in[p, u]$ and $b \in[q, u]$ with $d(a, u)=d(b, u)=\rho$, and let $\bar{a}$ and $\bar{u}$ be comparison points in $\bar{\Delta}$. Let $\bar{\theta}=\angle_{\bar{u}}(\bar{p}, \bar{q})$. Since $\bar{\theta} \geq \theta \geq \theta_{1}$, we must have $d(\bar{a}, \bar{b})=f(\bar{\theta}, \rho) \geq 2 \delta$. By the $\delta$-thinness of $\bar{\Delta}$, there exist $\bar{v}$ and $\bar{w}$ on $[\bar{p}, \bar{q}]$ such that $d(\bar{a}, \bar{v})$ and $d(\bar{b}, \bar{w})$ are both at most $\delta$ and such that $\bar{w}$ lies between $\bar{v}$ and $\bar{q}$. Let $v$ and $w$ be the points on $[p, q]$ corresponding to $\bar{v}$ and $\bar{w}$. Then $d(a, v)$ and $d(b, w)$ are at most $\delta$ and $w$ lies between $v$ and $q$. As $d(p, v) \geq d(p, a)-\delta$ and $d(q, w) \geq d(q, b)-\delta$ we get

$d(p, q) \geq d(p, v)+d(w, q) \geq d(p, a)+d(q, b)-2 \delta=d(p, u)+d(u, q)-2 \delta-2 \rho$,

as claimed. 


\section{References}

[1] U. Abresch and V. Schroeder, Graph manifolds, ends of negatively curved spaces and the hyperbolic 120-cell space. J. Differential Geom. 35 (1992), 299-336. Zbl 0777.53035 MR 1158338

[2] D. Allcock, Asphericity of moduli spaces via curvature. J. Differential Geom. 55 (2000), 441-451. Zbl 1067.53028 MR 1863730

[3] D. Allcock, J. A. Carlson, and D. Toledo, Orthogonal complex hyperbolic arrangements. In Symposium in Honor of C. H. Clemens (Salt Lake City, UT, 2000), Contemp. Math. 312, Amer. Math. Soc., Providence, RI, 2002, 1-8. Zbl 1044.32023 MR 1941572

[4] D. Allcock, J. A. Carlson, and D. Toledo, Addendum to [3]. http://www.ma.utexas.edu/users/allcock/research/hyperplanes_addendum.html

[5] M. T. Anderson, The Dirichlet problem at infinity for manifolds of negative curvature. $J$. Differential Geom. 18 (1983), 701-721 (1984). Zbl 0541.53036 MR 730923

[6] J. Behrstock, C. Druţu, and L. Mosher, Thick metric spaces, relative hyperbolicity, and quasi-isometric rigidity. Math. Ann. 344 (2009), 543-595. Zbl 1220.20037 MR 2501302

[7] I. Belegradek, Aspherical manifolds with relatively hyperbolic fundamental groups. Geom. Dedicata 129 (2007), 119-144. Zbl 1139.20036 MR 2353987

[8] I. Belegradek, Complex hyperbolic hyperplane complements. Math. Ann. 353 (2012), 545-579. Zbl 06043340 MR 2915548

[9] I. Belegradek, Rigidity and relative hyperbolicity of real hyperbolic hyperplane complements. Pure Appl. Math. Q. 8 (2012), 15-51. Zbl 1252.53042 MR 2900079

[10] G. Bell and A. Dranishnikov, On asymptotic dimension of groups acting on trees. Geom. Dedicata 103 (2004), 89-101. Zbl 1131.20032 MR 2034954

[11] G. C. Bell and A. N. Dranishnikov, A Hurewicz-type theorem for asymptotic dimension and applications to geometric group theory. Trans. Amer. Math. Soc. 358 (2006), 4749-4764. Zbl 1117.20032 MR 2231870

[12] B. H. Bowditch, Notes on Gromov's hyperbolicity criterion for path-metric spaces. In Group theory from a geometrical viewpoint (Trieste, 1990), World Sci. Publ., Singapore 1991, 64-167. Zbl 0843.20031 MR 1170364

[13] B. H. Bowditch, Discrete parabolic groups. J. Differential Geom. 38 (1993), 559-583. Zbl 0793.53029 MR 1243787

[14] B. H. Bowditch, Geometrical finiteness with variable negative curvature. Duke Math. J. 77 (1995), 229-274. Zbl 0877.57018 MR 1317633

[15] B. Bowditch, Relatively hyperbolic groups. Internat. J. Algebra Comput. 22 (2012), no. 3 , 1250016-1-1250016-66. Zbl 06042654 MR 2922380

[16] M. R. Bridson and A. Haefliger, Metric spaces of non-positive curvature. Grundlehren Math. Wiss. 319, Springer-Verlag, Berlin 1999. Zbl 0988.53001 MR 1744486

[17] J. L. Bryant, Piecewise linear topology. In Handbook of geometric topology, NorthHolland, Amsterdam 2002, 219-259. Zbl 0986.57021 MR 1886671

[18] I. Bumagin, The conjugacy problem for relatively hyperbolic groups. Algebr. Geom. Topol. 4 (2004), 1013-1040. Zbl 1111.20035 MR 2100689 
[19] J. Cheeger and D. Gromoll, On the structure of complete manifolds of nonnegative curvature. Ann. of Math. (2) 96 (1972), 413-443. Zbl 0246.53049 MR 0309010

[20] M. Coornaert, T. Delzant, and A. Papadopoulos, Géométrie et théorie des groupes. Lecture Notes in Math. 1441, Springer-Verlag, Berlin 1990. Zbl 0727.20018 MR 1075994

[21] F. Dahmani, Combination of convergence groups. Geom. Topol. 7 (2003), 933-963. Zbl 1037.20042 MR 2026551

[22] C. Druţu and M. Sapir, Tree-graded spaces and asymptotic cones of groups. Topology 44 (2005), 959-1058. Zbl 1101.20025 MR 2153979

[23] C. Druţu and M. V. Sapir, Groups acting on tree-graded spaces and splittings of relatively hyperbolic groups. Adv. Math. 217 (2008), 1313-1367. Zbl 1138.20024 MR 2383901

[24] D. B. A. Epstein, J. W. Cannon, D. F. Holt, S. V. F. Levy, M. S. Paterson, W. P. Thurston, Word processing in groups. Jones and Bartlett Publishers, Boston 1992. Zbl 0764.20017 MR 1161694

[25] B. Farb, Relatively hyperbolic groups. Geom. Funct. Anal. 8 (1998), 810-840. Zbl 0985.20027 MR 1650094

[26] K. Fujiwara, The second bounded cohomology of a group acting on a Gromov-hyperbolic space. Proc. London Math. Soc. (3) 76 (1998), 70-94. Zbl 0891.20027 MR 1476898

[27] M. Gromov, Volume and bounded cohomology. Inst. Hautes Études Sci. Publ. Math. 56 (1982), 5-99. Zbl 0516.53046 MR 686042

[28] M. Gromov, Hyperbolic groups. In Essays in group theory, Math. Sci. Res. Inst. Publ. 8, Springer-Verlag, New York 1987, 75-263. Zbl 0634.20015 MR 0919829

[29] M. Gromov, Geometric group theory (Sussex, 1991), vol. 2: Asymptotic invariants of infinite groups. London Math. Soc. Lecture Note Ser. 182, Cambridge University Press, Cambridge 1993. Zbl 0841.20039 MR 1253544

[30] J. Hempel, Residual finiteness for 3-manifolds. In Combinatorial group theory and topology (Alta, Utah, 1984), Ann. of Math. Stud. 111, Princeton University Press, Princeton, NJ, 1987, 379-396. Zbl 0772.57002 MR 895623

[31] J. Hempel, 3-manifolds. AMS Chelsea Publishing, Providence, RI, 2004. Zbl 1058.57001 MR 2098385

[32] C. Löh and R. Sauer, Degree theorems and Lipschitz simplicial volume for nonpositively curved manifolds of finite volume. J. Topol. 2 (2009), 193-225. Zbl 1187.53043 MR 2499443

[33] I. Mineyev and A. Yaman, Relative hyperbolicity and bounded cohomology. Preprint. http://www.math.uiuc.edu/ mineyev/math/art/rel-hyp.pdf

[34] D. Osin, Asymptotic dimension of relatively hyperbolic groups. Internat. Math. Res. Notices 2005 (2005), 2143-2161. Zbl 1089.20028 MR 2181790

[35] D. V. Osin, Peripheral fillings of relatively hyperbolic groups. Invent. Math. 167 (2007), 295-326. Zbl 1116.20031 MR 2270456

[36] G. P. Paternain and J. Petean, Minimal entropy and collapsing with curvature bounded from below. Invent. Math. 151 (2003), 415-450. Zbl 1049.53029 MR 1953264

[37] W. A. Poor, Jr., Some results on nonnegatively curved manifolds. J. Differential Geom. 9 (1974), 583-600. Zbl 0292.53037 MR 0375155 
[38] J.-P. Préaux, Conjugacy problem in groups of oriented geometrizable 3-manifolds. Topology 45 (2006), 171-208. Zbl 1088.57001 MR 2170497

[39] J.-P. Préaux, Conjugacy problem in groups of non-oriented geometrizable 3-manifolds. Preprint 2005. arXiv:math/0512484

[40] V. A. Sharafutdinov, Complete open manifolds of nonnegative curvature. Sibirsk. Mat. Ž. 15 (1974), 177-191; English transl. Siberian Math. J. 15 (1974), 126-136. Zbl 0288.53039 MR 0343208

[41] P. Tukia, Convergence groups and Gromov's metric hyperbolic spaces. New Zealand J. Math. 23 (1994), 157-187. Zbl 0855.30036 MR 1313451

Received March 18, 2010; revised September 14, 2011

I. Belegradek, School of Mathematics, Georgia Institute of Technology, Atlanta,

GA 30332-0160, U.S.A.

E-mail: ib@math.gatech.edu

G. C. Hruska, Department of Mathematical Sciences, University of Wisconsin-Milwaukee, P.O. Box 413, Milwaukee, WI 53201-0413, U.S.A.

E-mail: chruska@uwm.edu 